\title{
Post-Plyometric Exercise Hypotension and Heart Rate in Normotensive Individuals:
}

\section{Influence of Exercise Intensity}

\author{
Hamid Arazi" $^{* 1 \mathrm{ADEF}}$, PhD; Abbas Asadi ${ }^{2 \mathrm{CDEG}}$, MSc; Mehdi Rahimzadeh ${ }^{1 \mathrm{BCG}}$, BSc; Amir-Hossein Moradkhani ${ }^{1 \mathrm{BCG}}$, BSc
}

\author{
Authors' Affiliation: \\ 1. Faculty of Sport Sciences, Department \\ of Exercise Physiology, University of \\ Guilan, Rasht, Iran \\ 2. Rasht Branch, Islamic Azad University, \\ Rasht, Iran

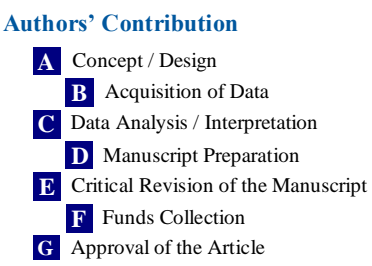

\section{* Corresponding Author;} \\ Address: Department of Exercise \\ Physiology, Faculty of Sport Sciences, \\ University of Guilan, Rasht, Iran \\ E-mail: hamidarazi@yahoo.com
}

Received: Oct 15, 2012

Accepted: May 25, 2013

Available Online: Jun 21, 2013

\begin{abstract}
Purpose: The purpose of this study was to compare the effects of high, moderate and low intensity plyometric exercise on the post-exercise systolic and diastolic blood pressure and heart rate responses.

Methods: Ten healthy normotensive men (age, $21.1 \pm 0.9$ years; height, $175.8 \pm 6$ $\mathrm{cm}$; and body mass, $69.1 \pm 13.6 \mathrm{~kg}$ ) volunteered to participate in this study and were evaluated for three non-consecutive days in depth jump exercise from $20-\mathrm{cm}$ box (low intensity [LI]), 40-cm box (moderate intensity [MI]) and $60-\mathrm{cm}$ box (high intensity $[\mathrm{HI}]$ ) for 5 sets of 20 repetitions. After each exercise session, systolic blood pressure (SBP), diastolic blood pressure (DBP) and heart rate (HR) were measured every 10 min for a period of 90 min.
\end{abstract}

Results: No significant differences were observed among post-exercise SBP, DBP and HR when the protocols (LI, MI and HI) were compared. The LI and HI protocols showed greater reduction in SBP at $40^{\text {th }}-\mathbf{7 0}{ }^{\text {th }}$ min of postexercise ( $\sim 9 \%)$, whereas the $\mathrm{LI}$ and $\mathrm{MI}$ protocols indicated greater reduction in DBP at $10^{\text {th }}-50^{\text {th }}$ min of post exercise $(\sim 10 \%)$. In addition, the change in the DBP for HI was not significant and the increases in the HR were similar for all intensities.

Conclusion: It can be concluded that a plyometric exercise (PE) can reduce SBP and DBP post-exercise and therefore we can say that PE has significant effects for reducing $B P$ and $H R$ or post-exercise hypotension.

Key Words: Plyometric; Systolic Blood Pressure; Diastolic Blood Pressure; Heart Rate

\section{INTRODUCTION}

$\mathrm{R}$ egular exercise has the potential to be a key nonpharmacological tool in the management of blood pressure (BP). After exercise, BP is normally reduced below pre-exercise level a well-known phenomenon termed "post-exercise hypotension (PEH)" ${ }^{[1]}$. PEH has been demonstrated after aerobic (endurance) exercise and resistance exercise ${ }^{[2,3]}$. In normotensive subjects, it has been attributed to a decrease in cardiac output and/or systemic vascular resistance ${ }^{[4,5]}$. Moreover, it has been accompanied by a decrease in peripheral sympathetic activity ${ }^{[5]}$, and an increase in cardiac sympathetic activity ${ }^{[6]}$. Nowadays, endurance and resistance exercise has also been recommended as part of a comprehensive exercise program to reduce cardiovascular risk in general population ${ }^{[7]}$, and in hypertensives ${ }^{[8]}$.

The benefits of endurance and resistance exercise on the cardiovascular system are well-documented. Evidence suggests that endurance and resistance exercise favorably alters resting arterial BP in persons with hypertension ${ }^{[8,9,10,11]}$. However, whether or not plyometric exercise results in a PEH response is relatively unknown. Plyometric exercise (PE) is one of the important types of training for the development of 
performance and is used in the conditioning schedule by athletes and conditioning coaches. Despite the important role of plyometric exercise, the information about this kind of exercise on the cardiovascular system, especially on BP is not completely understood. Only a few studies have investigated the BP responses following PE, and these have shown conflicting results $[12,13,14]$. Previous authors showed that PE can increase BP after each set of exercise ${ }^{[13,14]}$, but the information about the effects of PE on PEH is scarce and no study has examined this approach. If PE is to be used as a non-pharmacological intervention in the management of $\mathrm{BP}$, more knowledge is required about the different characteristics (i.e. number of sets and repetitions per set, rest between sets, intensity etc.) of the exercise required to evoke $\mathrm{PEH}$, especially the intensity of the bout. Because BP responses during the recovery period can be influenced by exercise intensity, it is possible that different exercise intensities may also have distinct effects on PEH.

Plyometric exercise intensity has been defined as the amount of stress the PE places on the muscle, connective tissue and joints ${ }^{[5]}$. During plyometric depth jump exercise, intensity is defined as the amount of landing force. With an increase in box height, the amount of intensity increases ${ }^{[15,16]}$. Although numerous studies have examined the effects of different resistance or endurance exercise intensities on BP and heart rate (HR) responses, limited data exist regarding the effects of different intensities of PE on PEH and HR responses. Since there were several differences between PE with resistance and endurance exercises, such as differences in mechanical nature and movement pattern; and it is more important to determine cardiovascular responses to this type of exercise, the current study was designed. Moreover, to our knowledge, no study has investigated the responses of BP and HR following PE. Therefore, the aim of the present study was to evaluate the effect of a single bout of different plyometric exercise intensities on BP and HR in normotensive subjects. Although PEH might have clinical relevance in hypertensives, the study was conducted with healthy subjects in order to understand its physiology without pathological influences. In this study, we had twofold purposes: (1) to determine if PE programs had beneficial effects on PEH and HR in male normotensive subjects; and (2) to compare acute bouts of low, moderate and high-intensity PE programs on PEH and HR.

\section{METHODS AND SUBJECTS}

\section{Subjects:}

Ten healthy normotensive men (age, $21.1 \pm 0.9$ years; height, $175.8 \pm 6 \mathrm{~cm}$; and body mass, $69.1 \pm 13.6 \mathrm{~kg}$ ), who were familiar with plyometric exercise and training, volunteered to participate in this study. The subjects were healthy, free from any lower body injuries and had no medical, cardiovascular and orthopedic problems that were confirmed by physician. The following exclusion criteria were observed: a) use of drugs that could influence cardiovascular responses at rest or during exercise, b) musculoskeletal limitations that would cause the exercises to be contraindicated and c) diagnosis of hypertension, heart disease or other cardiovascular problems that would cause the exercises to be contraindicated. Before data collection, the participants were informed about the benefit, and possible risks associated with the study and provided written informed consent. The participants did not change their sleeping, eating or drinking habits during the study process. This study was approved by the institutional ethical committee and was conducted according to the Declaration of Helsinki.

\section{Experimental design:}

The data collection was performed on four nonconsecutive days. At least 7 days prior to the experiments, subjects reported to laboratory for the measurement of age, body mass and height. During this session, each participant was instructed in the proper form and technique of depth jump exercise (familiarization session, day 1). All subjects underwent, in a random order, three experimental sessions: Low intensity (LI), Moderate intensity (MI) and High intensity (HI).

Sessions were initiated between 1 and 3 PM, and had an interval of at least 7 days. Subjects were 
instructed to take a light meal 2 hours before the experiments, to avoid physical exercise and alcohol for at least 48 hours before performing $\mathrm{PE}$, and to avoid smoking, caffeine, and medications for 12 hours. Before performing the protocols, the subjects performed a 10 min warm-up including light running, static stretching and ballistic movements, and then remained seated for $10 \mathrm{~min}$ in a calm and quiet environment. The HR and BP were then measured at rest (pre-intervention period). Then they moved to the exercise room, where they remained for about $20 \mathrm{~min}$ : LI depth jump exercise, MI depth jump exercise and HI depth jump exercise. The ambient temperature was fixed at $27 \pm 1 \mathrm{C}^{\circ}$ and the air humidity during the tests ranged between $60 \%$ and $70 \%$. Also after performing the protocols, subjects came back to laboratory and seated for $90 \mathrm{~min}$ in a quiet and comfortable place, to measure the post-exercise BP every $10 \mathrm{~min}$ (postintervention period). An experienced appraiser performed the measurements during rest and after exercise for all subjects.

\section{Plyometric exercise:}

After a 10 min standard warm-up, participants performed plyometric protocols in a random order. Each participant performed 5 sets of 20 repetitions of depth jump from the height of a 20-cm box (day 2, LI), 40-cm box (day 3, MI) and 60-cm box (day 4, HI) onto a wrestling-type mat with an interval of $8 \mathrm{sec}$ between jumps and $120 \mathrm{sec}$ between sets. Subjects began by standing on the plyometric box and were instructed to lead with one foot as they stepped down from the box and land with two feet on the ground. Instantly upon ground contact, subjects were instructed to explode off the ground by jumping as quickly and as high as possible. Subjects were instructed to perform each jump by maximal effort and proper technique. These exercise programs were based on recommendations of intensity from Miyama and Nosaka ${ }^{[17]}$ and Ebben et al [16].

\section{Blood pressure and heart rate measurements:}

Systolic blood pressure (SBP) and diastolic blood pressure (DBP) were measured by the indirect auscultatory method using a sphygmomanometer (Missouri $^{\circledR}$ ) and stethoscope (Rappaport ${ }^{\circledR}$ GF Health
Products, Northeast Parkway Atlanta). BP was assessed before the exercise bout and at $10 \mathrm{~min}$ intervals for 90 minutes after the exercise bout. During the measurements, the volunteers remained seated on a comfortable couch in an environment without noise. The HR was measured using Polar S610i heart rate monitor (FIN, 90440, FINLAND) (beat/min).

\section{Statistical analyses:}

Data are presented as mean \pm standard deviation. A 2way repeated-measures ANOVA followed by the Bonferroni post hoc test, where indicated, were used to analyze SBP, DBP and HR. The level of significance was set at $P \leq 0.05$ for all statistical procedures. All analyses were conducted using SPSS version 16.0 (SPSS Inc., Chicago, IL, USA).

\section{RESULTS}

No significant differences were observed among postexercise SBP, DBP and HR when the programs (LI, MI and $\mathrm{HI}$ ) were compared. All intensities indicated significant decreases in the post-exercise SBP level at $10^{\text {th }}, 20^{\text {th }}$ and $30^{\text {th }}$ min of post-exercise. Only LI and HI showed significant decreases in the SBP at $40^{\text {th }}-70^{\text {th }}$ min of post-exercise (Fig. 1).

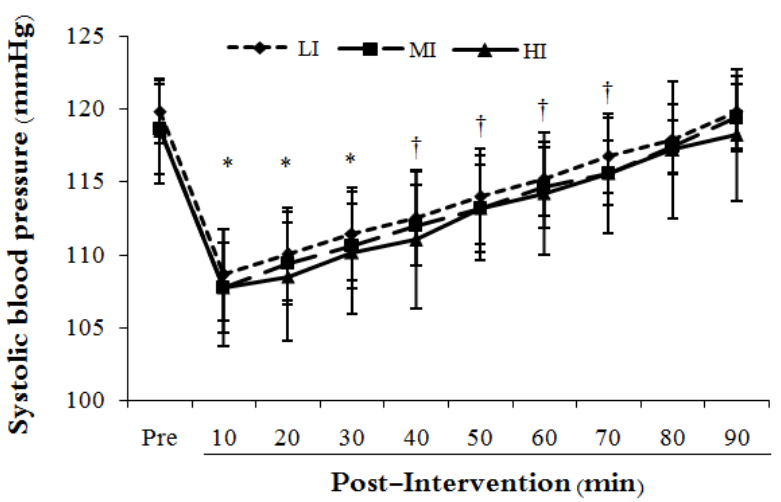

Fig. 1: Mean \pm SD of systolic blood pressure at pre-exercise and post performing low intensity (LI), moderate intensity (MI) and high intensity (HI) plyometric exercise. * Significant decreases $(P \leq 0.05)$ compared with pre-exercise value for all intensities, $\uparrow$ Significant decreases $(P \leq 0.05)$ compared with pre-exercise value for LI and HI. 


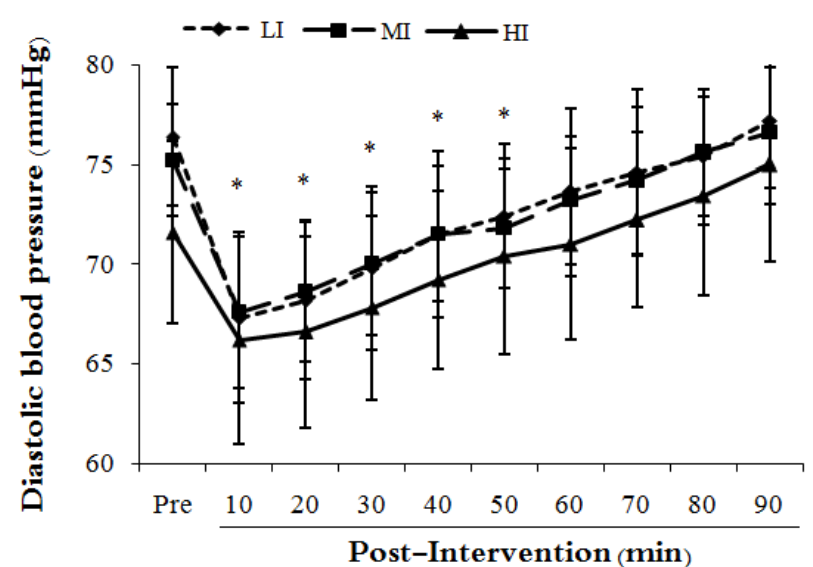

Fig. 2: Mean \pm SD of diastolic blood pressure at pre - exercise and post performing low intensity (LI), moderate intensity (MI) and high intensity (HI) plyometric exercise.

* Significant decreases $(P \leq 0.05)$ compared with pre-exercise value for LI and MI.

In the DBP, no significant changes were observed for the HI, whereas significant reduction was seen at $10^{\text {th }}$ $50^{\text {th }}$ min of post-exercise for the LI and MI (Fig. 2). All protocols induced significant increases in the postexercise HR at $10^{\text {th }}-50^{\text {th }}$ min of post-exercise. The HR returned at pre-exercise level at $70^{\text {th }} \mathrm{min}$ of postexercise. Significant reductions were also found for all intensities in the HR at $90^{\text {th }}$ min of post-exercise when compared with pre-exercise (Fig. 3).

\section{DISCUSSION}

Considering the important role of plyometric exercise and training for improving muscular performance, the effects of this exercise on cardiovascular responses is important and few studies have focused on this area and information about this aspect is little and scarce. Therefore, this study was designed to examine the effect of a bout of plyometric exercise with differing intensity on PEH and HR. In the present study, we observed that post exercise SBP was significantly reduced by all intensities $30 \mathrm{~min}$ post-protocol, but there were only significant reduction in the $40^{\text {th }}-70^{\text {th }}$ min after the LI and HI program. In addition, DBP was significantly reduced in $10^{\text {th }}-50^{\text {th }}$

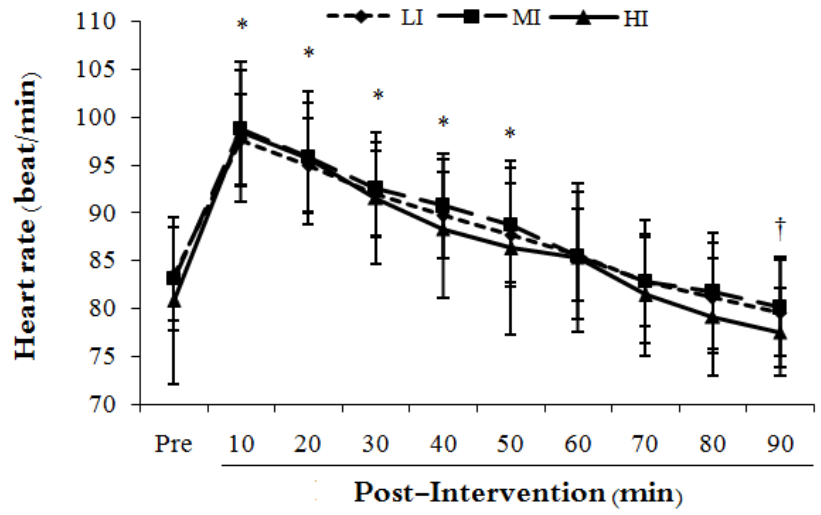

Fig. 3: Mean \pm SD of heart rate at pre-exercise and post performing low intensity (LI), moderate intensity (MI) and high intensity (HI) plyometric exercise.

* Significant increases $(P \leq 0.05)$ compared with pre-exercise value for all intensities.

$\dagger$ Significant decreases $(P \leq 0.05)$ compared with pre-exercise value for all intensities.

min after LI and MI programme, whereas no significant changes were observed after HI programme. In the HR, all intensities showed significant increases in the $10^{\text {th }}-50^{\text {th }}$ min after PE, and significantly reduced in the $90^{\text {th }}$ min after PE. The findings of the present study are in line with previous studies that investigated $\mathrm{PEH}$ after resistance and endurance exercises ${ }^{[2,13,18]}$. In contrast, Brown et al ${ }^{[9]}$ examined the effects of 8 sets of 10 reps of depth jump from $80 \mathrm{~cm}$ box on BP and HR responses, and found no changes in SBP, DBP at 5-10 min post exercise in normotensive men and women.

Several mechanisms have been proposed to account for the decreases in BP during recovery from PE. Both central and peripheral hemodynamic factors have been implicated as potential contributors to $\mathrm{PEH}^{[7]}$. A drop in peripheral resistance has been reported during recovery from aerobic activity and resistance exercise ${ }^{[2,3,8,19]}$. The fall in peripheral resistance may be related to thermoregulatory vasodilatation ${ }^{[21]}$, suppression in sympathetic nerve activity ${ }^{[19,22]}$ or to the release of vasodilator metabolites from skeletal muscle ${ }^{[1,9,23]}$. An accumulation of metabolites may be of particular interest with $\mathrm{PE}$, which is associated with a greater reliance on nonoxidative metabolic pathways. Hill and coworkers ${ }^{[23]}$ attributed a sudden dramatic drop in SBP immediately following heavy exercise to a reduction in venous return and the removal of mechanical resistance 
to blood flow and a subsequent lowering of peripheral resistance. The role of central hemodynamic variables in PEH has yet to be elucidated. Some researchers have reported a reduction in cardiac output and stroke volume following exercise ${ }^{[22,24]}$, whereas others have found an elevation of these parameters ${ }^{[19]}$. Rueckert et al ${ }^{[25]}$ observed a biphasic hemodynamic pattern characterized by a drop in peripheral resistance during the initial 20 min of recovery and a decrease in cardiac output from 50 to 120 min following exercise.

In this study, it is possible that PE induces a reduction in sympathetic nerve activity to the heart and blood vessels during the recovery period. Chen et al ${ }^{[26]}$ observed a decrease in sympathetic tone to the heart after mild exercise in spontaneously hypertensive rats and Floras et al ${ }^{[22]}$ and Halliwill et al ${ }^{[5]}$ found a significant decrease in muscle sympathetic nerve activity after acute exercise in hypertensive and normotensive humans. Previous authors observed that after an acute bout of exercise, baroreflex control of heart rate and blood vessels is distinctly regulated ${ }^{[5]}$. In other words, sympathetic drive to the heart increases, while sympathetic drive to blood vessels decreases. Thus, it is possible that different exercise intensities may distinctly affect the baroreflex control of heart rate, but not the baroreflex control of blood vessels. Moreover, the vasodilatory response ${ }^{[27]}$ and/or the decrease in blood volume ${ }^{[28]}$ may play a role in the post-exercise blood pressure fall. It is well understood that muscle metabolites and heat accumulation are directly related to exercise intensity, and sweating rate is greater during intense exercise ${ }^{[29]}$. In addition, the increased local muscle metabolites ${ }^{[20]}$ and/or heat production ${ }^{[5]}$ are also potential stimuli for the increased heart rate responses after PE. On the other hand, a decrease in muscle cell $\mathrm{pH}$ following PE may stimulate chemosensitive afferent fibers, thereby elevating HR ${ }^{[30]}$. The forces and intensity of PE [31], greater involvement of the fast-twitch muscle fibers ${ }^{[18]}$, and size of activated muscle mass ${ }^{[32]}$ may also stimulate increases in HR and BP.

Overall, according to findings of present study the mechanism (s) causing decreases in BP and increases in $\mathrm{HR}$ can be increases in $\mathrm{pH}$, increase in muscle metabolites and heat production after plyometric exercise. Although, the present investigation was the first study about the post plyometric exercise hypotension, more studies are needed to define the role of plyometric exercise in management of BP in human subjects.

\section{CONCLUSION}

In summary, we found that plyometric exercise induced significant reduction in BP, and increases in HR. These findings are in line with previous studies that used resistance and endurance exercises. According to results of this study we can say that PE has significant effects for deceasing BP after exercise. Although we cannot directly recommend PE for decreasing BP, these results show significant decreases in $\mathrm{BP}$ after $\mathrm{PE}$ and this finding necessitates more studies.

\section{ACKNOWLEDGMENTS}

The authors gratefully acknowledge all the subjects who took part in this study for their cooperation.

Conflict of interests: None

\section{REFERENCES}

[1] Kenny MJ, Seals DR. Postexercise hypotension. Key features, mechanisms and clinical significant. Hypertension 1993; 22:65364.

[2] Boroujerdi SS, Rahimi R, Noori SR. Effects of high versus low intensity resistance training on post-exercise hypotension in male athletes. Int Sport Med J 2009:10;95-100.

[3] Mohebbi H, Rahmaninia F, Sheikholeslami vatani D, Faraji H. Post-exercise responses in blood pressure, heart rate and rate pressure product in endurance and resistance exercise. Med dell Sport 2010;63:209-19. 
[4] Forjaz CL, Cardoso CG JR, Rezk CC, et al. Postexercise hypotension and hemodynamics: the role of exercise intensity. J Sports Med Phys Fitness 2004;44:54-62.

[5] Halliwill JR, Taylor JA, Eckberg DL. Impaired sympathetic vascular regulation in humans after acute dynamic exercise. $J$ Physiol 1996;495:279-88.

[6] Piepoli M, Coats AJ, Adamopoulos S, et al. Persistent peripheral vasodilation and sympathetic activity in hypotension after maximal exercise. J Appl Physiol 1993;75:1807-14.

[7] American College of Sports Medicine. ACSM's guidelines for exercise testing and prescription. Lippincott Williams and Wilkins, Philadelphia; 2000.

[8] Pescatello LS, Franklin BA, Fagard R, et al. American College of Sports Medicine position stand. Exercise and hypertension. Med Sci Sports Exerc 2004;36:533-53.

[9] Brown SP, Clemons JM, He Q, Liu S. Effects of resistance exercise and cycling on recovery blood pressure. J Sports Sci 1994; $12: 463-8$.

[10] O'Connor PJ, Bryant CX, Veltri JP, Gebhardt SM. State anxiety and ambulatory blood pressure following resistance exercise in females. Med Sci Sports Exerc 1993;25:516-21.

[11] MacDonald JR, MacDougall JD, Interisano SA, et al. Hypotension following mild bouts of resistance exercise and submaximal dynamic exercise. Eur J Appl Physiol Occup Physiol 1999;79:148-54.

[12] Roltsch MH, Mendez T, Wilund KR, Hagberg JM. Acute resistive exercise does not affect ambulatory blood pressure in young men and women. Med Sci Sports Exerc 2001;33:881-6.

[13] Arazi H, Asadi A, Nasehi M, Delpasand A. Cardiovascular and blood lactate responses to acute plyometric exercise in female volleyball and handball players. Sport Sci Health 2012;8:23-9.

[14] Brown GA, Ray MW, Abbey BM, et al. Oxygen consumption, heart rate, and blood lactate responses to an acute bout of plyometric depth jumps in college-aged men and women. J Strength Cond Res 2010;24(9):2475-82.

[15] Ebben WP, Simenz C, Jensen R. Evaluation of plyometric intensity using electromyography. J Strength Cond Res 2008;22:8618.

[16] Ebben WP. Practical guidelines for plyometric intensity. Nat Strength Cond Asso 2007;6:12-6.

[17] Miyama M, Nosaka K. Influence of surface on muscle damage and soreness induced by consecutive drop jumps. J Strength Cond Res 2004;18:206-21.

[18] Collins MA, Cureton KJ, Hill DW, Ray CA. Relationship of heart rate to oxygen uptake during weight lifting exercise. Med Sci Sports Exerc 1991;23:636-40.

[19] Cle'roux J, Kouame N, Nadeau A, et al. After effects of exercise on regional and systemic hemodynamics in hypertension. Hypertension 1992;19:183-91.

[20] Middlekauff HR, Nitzsche EU, Nguyen AH, Hoh CK. Modulation of renal cortical blood flow during static exercise in humans. Circulation Res 1997;80:62-68.

[21] Franklin PJ, Green DJ, Cable NT. The influence of thermoregulatory mechanisms on post-exercise hypotension in humans. $J$ Physiol 1993;470:231-41.

[22] Flores JS, Sinkey CA, Aylward PE, et al. Postexercise hypotension and sympathoinhibition in borderline hypertensive men. Hypertension 1989;14:28-35.

[23] Hill DW, Collins MA, Cureton KJ, Demello JJ. Blood pressure response after weight training exercise. J Appl Sport Sci Res 1989;3:44-47.

[24] Hagberg JM, Montain SJ, Martin WH. Blood pressure and hemodynamic responses after exercise in older hypertensives. $J$ Appl Physiol 1987;63:270-76.

[25] Rueckert PA, Slane PR, Lillis DL, Hanson P. Hemodynamic patterns and duration of post dynamic exercise hypotension in hypertensive humans. Med Sci Sports Exerc 1996;28:24-32.

[26] Chen Y, Chandler MP, DiCarlo SE. Acute exercise attenuates cardiac autonomic regulation in hypertensive rats. Hypertension 1995;26:676-83.

[27] Piepoli M, Isea JE, Pannarale G, et al. Load dependence of changes in forearm and peripheral vascular resistance after acute leg exercise in man. J Physiol 1994;478:357-62.

[28] Overton JM, Joyner MJ, Tipton CM. Reductions in blood pressure after acute exercise by hypertensive rats. J Appl Physiol $1988 ; 64: 748-52$.

[29] McArdle WD, Katch FI, Katch VL. Exercise and thermal stress. In: Exercise Physiology: Energy, Nutrition, and Human Performance. Williams \& Wilkins, Baltimore, MD; 1996.

[30] Victor RG, Bertocci LA, Pryor SL, Nunally RL. Sympathetic discharge is coupled to muscle cell pH during exercise in humans. J Clin Invest 1988;82:1301-05.

[31] Keul J, Haralamble G, Bruder M, Gottstein HJ. The effect of weight lifting exercise on heart rate and metabolism in experienced weight lifters. Med Sci Sports Exerc 1978;10:13-5.

[32] Rozenek R, Rosenau L, Rosenau P, Stone MH. The effect of intensity on heart rate and blood lactate response to resistance exercise. J Strength Cond Res 1993; 7:51-4. 